\title{
Evaluating DNA methylation age on the Illumina's methylationEPIC BeadChip
}

2 Dhingra, Radhika 1,2,3*, Lydia Coulter Kwee ${ }^{4}$, David Diaz-Sanchez ${ }^{1}$, Robert B. Devlin ${ }^{1}$, Wayne

3 Cascio $^{1}$, Carol Haynes ${ }^{2}$, Elizabeth R. Hauser ${ }^{4,5,6}$, Simon Gregory ${ }^{6}$, Svati Shah ${ }^{4,7}$, William

4 Kraus $^{4,7}$, Kenneth Olden ${ }^{8}$, Cavin K. Ward-Caviness ${ }^{1}$

$5 \quad{ }^{1}$ National Health and Environmental Effects Laboratory, US Environmental Protection Agency, Chapel Hill, NC, USA

${ }^{2}$ Department of Environmental Sciences and Engineering, Gillings School of Public

${ }^{3}$ Institute for Environmental Health Solutions, University of North Carolina, Chapel Hill, NC USA

${ }^{4}$ Duke Molecular Physiology Institute, Duke University Medical Center, Durham, NC, USA

${ }^{5}$ Department of Biostatistics and Bioinformatics, Duke University Medical Center, Durham, NC, USA

${ }^{6}$ Cooperative Studies Program Epidemiology Center, Durham Veterans Affairs Medical Center, Durham, NC, USA

${ }^{7}$ Division of Cardiology, Department of Medicine, School of Medicine, Duke University, Durham, NC, USA

${ }^{8}$ National Center for Environmental Assessment, US Environmental Protection Agency, Chapel Hill, NC, USA

*Corresponding Author: Radhika Dhingra

22 Disclaimer: This paper has been reviewed by the National Health and Environmental Effects

23 Research Laboratory, U.S. EPA, and is approved for publication. Approval does not signify that

24 the contents necessarily reflect the views and policies of the agency, nor does mention of trade

25 names or commercial products constitute endorsement or recommendation for use. The authors

26 declare they have no competing financial interests. 
Abstract

DNA methylation age (DNAm age) has become a widely utilized epigenetic biomarker

29 for the aging process. The Horvath method for determining DNAm age is perhaps the most

30 widely utilized and validated DNA methylation age assessment measure. Horvath DNAm age is

31 calculated based on methylation measurements at 353 loci which were present on Illumina's

$32450 \mathrm{k}$ and 27k DNA methylation microarrays. With the increasing use of the more recently

33 developed Illumina MethylationEPIC (850k) microarray, it is worth revisiting this widely used

34 aging measure to evaluate differences in DNA methylation age estimation based on array design.

35 Of the requisite 353 loci, 17 are missing from the current 850k microarray. Using 17 datasets

36 with $27 \mathrm{k}, 450 \mathrm{k}$, and/or 850k methylation data, we calculated and compared each sample's

37 epigenetic age estimated from all 353 loci required from the Horvath DNAm age calculator

38 (full), and using only the 336 loci present on the $27 \mathrm{k}, 450 \mathrm{k}$, and $850 \mathrm{k}$ arrays (reduced). In

$39450 \mathrm{k} / 27 \mathrm{k}$ data, missing loci caused underestimation of epigenetic age when compared with the

40 full clock. Underestimation of full epigenetic age grew from ages 0 to $\sim 20$, remaining stable

41 thereafter (mean $=-3.46 \mathrm{y}, \mathrm{SD}=1.13$ ) years for individuals $\geq 20$ years. Underestimation of DNAm

42 age by the reduced $450 \mathrm{k} / 27 \mathrm{k}$ data was similar to the underestimation observed in the $850 \mathrm{k}$ data

43 indicating that array differences in DNAm age estimation are primarily driven by missing

44 probes. Correlations between age and DNAm age were not dependent on missing probes or on

45 array designs and consequently associations between DNAm age and outcomes such as sex

46 remained the same independent of missing probes and probe design. In conclusion, DNAm age

47 estimations are array dependent driven by missing probes between arrays. Though correlations

48 and associations with DNAm age may remain the same, researchers should exercise caution

49 when interpreting results based on absolute differences in DNAm age or when mixing samples

50 assayed on different arrays. 


\section{Introduction}

DNA methylation has recently shown promise as a potentially clinically useful biomarker of aging. A recent "epigenetic clock" developed by Horvath (1) has been shown to be an accurate estimator of age across multiple tissues and populations, and differences between DNA methylation age and chronological age are associated with pathophysiological biomarkers and incident disease (2).

The method developed by developed by Horvath (1) is perhaps the most widely used and validated epigenetic age estimation method; it relies on measurement of percent methylation at 353 loci (CpGs) on either the Illumina 450k (450k) or Illumina 27k (27k) microarray chips. Recently, Illumina released the Infinium MethylationEPIC Bead Chip (850k), which uses the same technology as the Illumina $450 \mathrm{~K}$ microarray to assay $866,836 \mathrm{CpGs}$ (3). Though the $850 \mathrm{k}$ microarray assays more loci, $8.9 \%$ of CpGs included on $450 \mathrm{~K}$ microarray were omitted from the $850 \mathrm{k}$ microarray. In particular, 17 of the $353 \mathrm{CpGs}(4.8 \%)$ necessary to calculate epigenetic age via the Horvath method are missing. While missing CpGs are imputed in the online calculator (4) to allow for estimation of epigenetic age, these missing probes may systematically bias the estimation of DNA methylation age and consequently alter the detection or interpretation of associations with health outcomes and inhibit cross-platform comparisons and analyses.

To evaluate the impact of microarray design changes on the estimation of DNA methylation age, we compared the Horvath DNA methylation age (DNAm age) calculated using all 353 CpGs (full DNAm age) to estimates obtained from using either the $27 \mathrm{k}$ or $450 \mathrm{k}$ platform while restricting to the $336 \mathrm{CpGs}$ available on the $850 \mathrm{k}$ platform. We used 15 publicly available non-cancer blood tissue datasets (available in the Gene Expression Omnibus(GEO),

73 https://www.ncbi.nlm.nih.gov/geo/), as well as blood samples from a cardiac catheterization cohort (CATHeterization GENetics; CATHGEN) where DNA methylation was assessed on both the $450 \mathrm{k}$ and $850 \mathrm{k}$ arrays.

\section{Methods}

\section{Missing loci and datasets}

To determine which loci in Horvath's original epigenetic clock loci are missing from the $850 \mathrm{k}$ platform we compared the $850 \mathrm{k}$ manifest of probe loci and the list of loci required for 
From the 81 datasets used to develop the Horvath epigenetic clock, we selected those 15 datasets (detailed in Supp. Table 1) whose non-cancerous samples were drawn from blood

83 (excluding cord blood), were publicly available on the Gene Expression Omnibus (GEO;

84 https://www.ncbi.nlm.nih.gov/geo//) and whose methylation beta values were readily available on

85 GEO. Though chronological age was not available in GSE42865 and GSE35069, and sex was not available in GSE30870 and GSE 42865, these datasets were also included in analyses that did not require age or sex.

Samples $(\mathrm{N}=3,672)$ in the 15 eligible GEO datasets (summarized in Table S1) were drawn from people ages 0 to 101, and included whole blood, peripheral blood monocytes (PBMC) and single leukocyte cell types. GSE 19711 was divided into two datasets (controls and ovarian cancer cases) for consistency with the Horvath epigenetic clock manuscript (1). Though

92 a few of these datasets include samples from cancer patients, the tissue obtained was non-

93 cancerous, and their methylation age had previously shown no association to cancer (1). Further

94 information about these datasets may be found on GEO, and in Additional file 2 of Horvath's

95 manuscript which describes these datasets and their rationale for inclusion in the development of 96 his epigenetic clock (1).

In addition to the GEO datasets, two datasets from the Catheterization Genetics cohort (CATHGEN) were employed to compare the $450 \mathrm{k}$ and $850 \mathrm{k}$ platforms. CATHGEN participants

99 were recruited from subjects undergoing an outpatient cardiac catheterization at Duke University

100 from 2001-2011 (5). Ethics approval was administered by the Duke Institutional Review Board 101 for CATHGEN.

The samples were processed by reading in the idat files using minfi v1.21.1, examining 103 samples for exclusion based on Illumina's default quality control (QC) procedures, background 104 correction via minfi's ssNoob, and extracting the un-normalized beta values. The CATHGEN 105 samples processed on the 450k and 850k microarrays were not obtained from the same 106 individuals, and no samples were excluded based on QC for the 450k microarray, while two 107 samples from the 850k microarray were excluded. This left 205 CATHGEN samples for the 108 450k microarray (ages 23-91 y) and 568 samples available from the 850k microarray (ages 33-87 109 y). 
Methylation beta values were extracted from the downloaded GEO datasets, and were not

112 further normalized before uploading to the (online) DNA Methylation Age Calculator as

113 recommended (https://dnamage.genetics.ucla.edu/). Where GEO datasets were previously

114 normalized, we deselected the normalize data option during processing in the DNA methylation

115 calculator; otherwise, the normalize data option was selected for unnormalized data.

116 All samples were included from the publicly available data. Sex, age, sample id and

117 blood type were extracted from the downloaded GEO datasets. The online DNA methylation age

118 calculator automatically imputes any missing probes

119 (https://labs.genetics.ucla.edu/horvath/dnamage/).

The epigenetic clock across the age ranges in 450k/27k data

To ascertain how the 17 missing loci might systematically misestimate epigenetic age via

122 Horvath's 353-probe DNA methylation clock, we calculated DNA methylation age in 27k and

123 450k datasets (GEO \& CATHGEN 450K datasets) with and without the 17 probes unavailable

124 on the 850k microarray. For each GEO dataset, as well as the CATHGEN 450k datasets, DNAm

125 age calculated using the reduced 450k data were compared to DNAm age calculated using the

126 full 450k data, graphically and using summary statistics. The comparisons were repeated in

127 subjects chronologically aged $20 \mathrm{y}$ or less, and in ages $\geq 20 \mathrm{y}$, a cutoff selected based on the

128 observed inflection point in the plot of age vs the difference in DNA methylation age estimated

129 using the full and reduced $450 \mathrm{k}$ data.

We hypothesized that the relationship of DNA methylation age to chronological age

131 differed in the full and reduced $450 \mathrm{k} / 27 \mathrm{k}$ datasets and that the difference varied by chronological 132 age group ( $>20$ years and $\leq 20$ years). Using all samples within each age group, we separately

133 regressed full 450k DNAm age and the reduced 450k DNAm age on chronological age, and

134 compared resulting the intercepts and chronological age slopes estimates. This analysis excluded

135 the GSE42865 and GSE35069 datasets as chronological age was not publicly available.

136 Within each age group, we also assessed the possibility that the relationship between

137 DNA methylation age, and thus age acceleration, and a clinical or other variable of interest could 138 be modified by the loss of 17 missing loci from the dataset. As sex was the only widely available 
139 variable in the public data, we separately regressed age acceleration estimated based on the full

140 and reduced 450k data on sex (ref. = Male), using all available samples within each age group.

141 We repeated these analyses in each individual dataset, without regard to the chronological age of

142 samples. We then statistically compared the slope obtained when using full 450k data age

143 acceleration to that obtained via reduced 450k data age acceleration for models of the association

144 of sex with age acceleration. Additionally, we compared residual plots of full and reduced 450k

145 data DNAm age acceleration regressed on chronological age for all GEO datasets where age was

146 available in the CATHGEN 450k dataset.

148 The CATHGEN data were used to ascertain if technological changes in the 850k

149 platform as compared to the $450 \mathrm{k}$ or $27 \mathrm{k}$ platforms contribute to mis-estimation of epigenetic

150 age. To that end, full and reduced datasets for the samples processed on the 450k, as well as a

151 dataset for the samples processed on the 850k were created for CATHGEN. Linear fits of the

152 epigenetic age by chronological age for each of the 3 CATHGEN datasets were produced. The

153 intercept and slopes of these linear fits were compared, to ascertain if the 850k platform impacts

154 the methylation measurement such that it would impact the calculation of epigenetic age, in a

155 manner separate from the effect of the 17 missing probes.

156 The CATHGEN dataset affords the ability to quantify any deviation of 850k DNAm ages 157 from expected values. As no 'correct' estimate of DNAm age on the 850k is available, we chose 158 regressed DNAm age on categorical variables for dataset types (full 450k and 850k in one model 159 and reduced $450 \mathrm{k}$ and $850 \mathrm{k}$ in the second model) while controlling for age. In both models, the 160 450k DNAm age, either full or reduced" was the referent category.

\section{Software and statistical analyses}

162 All work to determine the lost loci, to prepare data for the online DNA Methylation Age

163 Calculator (https://dnamage.genetics.ucla.edu/) and to subsequently compare epigenetic age

164 estimates with chronological age were performed in R (version 3.4.0) (6).

\section{Terminology}

166 Three categories of DNA methylation data were used in this analysis: 1) data from the Illumina $167450 \mathrm{k}$ array or the $27 \mathrm{k}$ array ("full 450k data"); 2) data from the Illumina 450k or 27k arrays with 
168 the 17 probes not on the Illumina 850k array removed ("reduced 450k data); and 3) data from the

169 Illumina 850k array ("850k data"). "Reduced 450k DNAm age" and "full 450k DNAm age"

170 refer to the application of the Horvath epigenetic clock to reduced and full 450k data,

171 respectively.

$172 \quad$ Results

173 Missing probes \& descriptions of the datasets

174 The 17 required DNA methylation age loci that are not included in the 850k manifest are

175 listed in Table 1. The GEO and CATHGEN 450k datasets together encompass 3,973 individuals

176 (52\% female, among those reporting sex) whose ages range from 0 (i.e., newborn) to 101 years

177 (Table 2). In addition, we had 568 independent CATHGEN samples that were processed on the

$178 \quad 850 \mathrm{k}$ platform.

179 Table 1. Missing probes, SNP presence, and symbol.

\begin{tabular}{ll}
\hline CpG & SNP? Symbol \\
\hline cg19945840 no & B3GALT6 \\
cg02972551 no & JMJD1A \\
cg02654291 yes & C9orf64 \\
cg13682722 yes & C14orf102 \\
cg09869858 yes & P11 \\
cg06117855 yes & CLEC3B \\
cg05590257 yes & LOC201164 \\
cg27016307 yes & HRC \\
cg24471894 yes & KIAA0020 \\
cg04431054 no & LOC133619 \\
cg16494477 no & FGF18 \\
gg19046959 no & COL8A2 \\
gg17408647 yes & FLJ10803 \\
gg27319898 no & FLJ32110 \\
gg19569684 no & PACAP \\
gg19273182 no & PAPOLG \\
$\mathbf{c g 0 9 7 8 5 1 7 2 ~ n o ~}$ & WFS1 \\
\hline & \\
\hline
\end{tabular}

180 
184 and CATHGEN datasets. The mean, standard deviations and correlation with chronological age (Age corr.) of DNAm age are

185 provided for each dataset.

\begin{tabular}{|c|c|c|c|c|c|c|c|c|c|}
\hline \multirow[b]{3}{*}{ GEO Series no. } & \multirow{3}{*}{$\begin{array}{l}\text { Plat- } \\
\text { form }\end{array}$} & \multirow{3}{*}{$\begin{array}{c}\mathrm{N} \text { (prop. } \\
\text { female) }\end{array}$} & & & \multicolumn{2}{|c|}{$\begin{array}{c}\text { (Full) 450k data } \\
\text { (353 loci) }\end{array}$} & \multicolumn{2}{|c|}{$\begin{array}{l}\text { Reduced 450k or 850k data } \\
\text { (336 loci) }\end{array}$} & \multirow{3}{*}{$\begin{array}{c}\text { Comparison } \\
\frac{\text { (450k data DNAm age) - }}{\text { (red. 450k data DNAm age })} \\
\text { Mean (SD) } \\
\end{array}$} \\
\hline & & & \multicolumn{2}{|c|}{$\underline{\text { Chronological age }}$} & \multicolumn{2}{|c|}{ DNA methylation age } & \multicolumn{2}{|c|}{ DNA methylation age } & \\
\hline & & & Median (range) & Mean (SD) & Mean (SD) & Age corr. & Mean (SD) & Age corr. & \\
\hline GSE19711 cases $(7,8)$ & $27 \mathrm{~K}$ & $266(1.0)$ & $67(49,91)$ & $66.42(9.35)$ & $62.5(11.47)$ & 0.55 & $58.43(11.02)$ & 0.56 & $4.11(0.81)$ \\
\hline GSE19711 controls $(7,8)$ & $27 \mathrm{~K}$ & $274(1.0)$ & $64(52,78)$ & $64.89(6.74)$ & $62.57(7.65)$ & 0.66 & $58.56(7.52)$ & 0.66 & $4.01(0.68)$ \\
\hline $\operatorname{GSE20067}(7,9)$ & $27 \mathrm{~K}$ & $192(0.51)$ & $43(24,74)$ & $43.9(9.8)$ & $43.45(9.27)$ & 0.81 & $38.55(9.2)$ & 0.81 & $4.85(0.95)$ \\
\hline GSE20236 (10) & $27 \mathrm{~K}$ & $93(1.0)$ & $63(49,74)$ & $62.86(6.33)$ & $53.79(6.51)$ & 0.69 & $49.92(6.32)$ & 0.68 & $3.87(0.58)$ \\
\hline GSE20242 (10) & $27 \mathrm{~K}$ & $50(0.74)$ & $34(16,69)$ & $35.86(13.89)$ & $45.02(27.45)$ & 0.55 & $41.49(27.71)$ & 0.53 & $2.30(0.84)$ \\
\hline GSE27097 (11) & $27 \mathrm{~K}$ & $398(0.0)$ & $9.3(3.6,17.8)$ & $9.89(3.63)$ & $9.6(4.41)$ & 0.75 & $8.14(3.88)$ & 0.72 & $1.46(0.69)$ \\
\hline $\operatorname{GSE30870}(12)^{* *}$ & $450 \mathrm{~K}$ & $38(0.74)$ & $44.5(0,100)$ & $46.32(47.01)$ & $41.06(42.02)$ & 0.99 & $38.93(39.95)$ & 0.99 & $2.14(2.13)$ \\
\hline GSE32149 (13) & $450 \mathrm{~K}$ & $48(0.52)$ & $15(3.5,76)$ & $22.15(18.43)$ & $22.3(15.13)$ & 0.96 & $19.96(14.34)$ & 0.97 & $2.34(0.92)$ \\
\hline GSE35069 (14)* & $450 \mathrm{~K}$ & $60(0.0)$ & NA & NA & $41.74(12.75)$ & - & $39.15(12.84)$ & - & $2.59(0.56)$ \\
\hline GSE36064 (11) & $450 \mathrm{~K}$ & $78(0.0)$ & $3.1(1.0,16.1)$ & $4.58(4.11)$ & $4.38(3.92)$ & 0.93 & $3.62(3.27)$ & 0.93 & $0.76(0.66)$ \\
\hline GSE40279 (15) & $450 \mathrm{~K}$ & $656(0.52)$ & $65(19,101)$ & $64.04(14.74)$ & $63.08(11.53)$ & 0.91 & $60.67(11.66)$ & 0.92 & $2.41(0.70)$ \\
\hline GSE41037 (16) & $27 \mathrm{~K}$ & $720(0.38)$ & $33(16,88)$ & $37.4(15.72)$ & $36.85(15.38)$ & 0.95 & 33.07 (15.07) & 0.96 & $3.81(0.79)$ \\
\hline GSE41169 (16) & $450 \mathrm{~K}$ & $95(0.29)$ & $29(18,65)$ & $31.57(10.28)$ & $31.23(11.01)$ & 0.94 & $27.67(10.69)$ & 0.94 & $3.55(0.60)$ \\
\hline GSE42861 (17) & $450 \mathrm{~K}$ & $689(0.71)$ & $54(18,70)$ & $51.93(11.8)$ & $53.38(11.09)$ & 0.90 & $50.22(11.01)$ & 0.90 & $3.16(0.58)$ \\
\hline GSE42865 $(18) * * *$ & $450 \mathrm{~K}$ & $15(0.62)$ & NA & NA & $38.19(9.45)$ & - & $35.68(9.68)$ & - & $2.40(1.10)$ \\
\hline CATHGEN 450k ${ }^{\circ}$ & $450 \mathrm{k}$ & $206(0.37)$ & $64(33,87)$ & $63.41(11.85)$ & $64.58(10.50)$ & 0.88 & $60.73(10.23)$ & 0.87 & $3.85(0.72)$ \\
\hline CATHGEN 850k $\uparrow^{\circ}$ & $850 \mathrm{k}$ & $568(0.41)$ & $59(23,91)$ & $60.11(12.44)$ & - & - & $58.16(10.51)$ & 0.86 & - \\
\hline
\end{tabular}

* As chronological age was missing for these datasets, correlation with age and age acceleration could not be determined.

** Proportion Female was obtained from supplemental table of the original epigenetic clock manuscript (Horvath, 2013), and were not available in GEO.

$\dagger$ Because the 17 loci required to complete the epigenetic clock are unavailable on the 850k platform, there is not information for the full epigenetic clock

${ }^{\circ}$ CATHGEN 450k and CATHGEN 850k are not comprised of the same individuals. That is, the underlying sample population is non-overlapping. 


\section{Comparison of DNA methylation age in 450k data and 850k data}

DNAm age estimated separately in the CATHGEN's full 450k, reduced 450k and 850k datasets using the epigenetic clock all showed positive correlations with chronological age (Table 2, Figure 1). For each of these three datasets, the slope between DNAm age and chronological age is nearly identical (0.73-0.78). However, in a regression of DNAm age on dataset type (full 450k vs. 850k) correcting for age, 850k DNAm ages had a mean difference of 3.96 y $(95 \% \mathrm{CI}:-4.08,-3.12 ; \mathrm{p}<0.0001)$ as compared to the full $450 \mathrm{k}$, which is very close to the underestimation seen with the when comparing CATHGEN DNAm age estimates from the reduced $450 \mathrm{k}$ data with the full $450 \mathrm{k}$ data (paired t-test: $3.85 \mathrm{y}, \mathrm{p}<0.0001$ ). There was no significant difference between the 850k DNAm age and reduced 450k DNAm age in CATHGEN $(-0.14 ; 95 \% \mathrm{CI}:-0.98,0.70, \mathrm{p}=0.75)$.

\section{Figure 1. Epigenetic age by chronological age in combinations of CATHGEN dataset and} epigenetic clock: The plot of DNA methylation by chronological age shows the impact of the 17 missing probes, by applying the epigenetic clock to CATHGEN 450k ('full' and 'reduced') and $850 k$ datasets.

\section{Probe exclusion effects on Horvath DNAm age in 16 datasets}

Across all 16 datasets with 450k or 27k data, reduced 450k DNAm age underestimated DNA methylation age as compared to the full 450k DNAm age (Figure S1). In peripheral blood samples from the youngest individuals (chronological age $<20 \mathrm{y}$ ), the individual difference between epigenetic age as estimated using the full and reduced datasets increased with age (Figure 2, Table 3). However, in samples from older individuals, (chronological age $\geq 20 \mathrm{y}$ ), the difference did not increase with age but we observed greater inter-individual variability in the difference between full and reduced DNAm age in older individuals $(\mathrm{SD}=1.13)$ than in the younger age group (ages 0-5y: $\mathrm{SD}=0.27$; ages 5-10y: 0.35; ages $10-15 \mathrm{y}: \mathrm{SD}=0.54$; and ages 15-20y: $\mathrm{SD}=0.82$ ). Across all datasets, the correlation between full and reduced 450k data remained high ranging from 0.989 to 0.999 .

\section{Figure 2. Difference of 'full' and 'reduced' epigenetic Age by chronological age. The}


difference of 'full and reduced' epigenetic ages calculated in the GEO (450k and 27k) and

220 CATHGEN 450k data are presented as (a) boxplot by 5 year chronological age categories and

221 (b) as a scatterplot.

Table 3. Regression of DNA methylation age on chronological age, by age group, in the full

224 and reduced $450 k / 27 k$ datasets (GEO and CATHGEN).

\begin{tabular}{lcccc}
\hline & \multicolumn{2}{c}{ Age $<20$ years (N=616) } & \multicolumn{2}{c}{ Age $\geq 20$ years (N =2.972) } \\
Intercept & Chronological Age & Intercept & Chronological Age \\
Data & Estimate (95\% CI) & Estimate (95\% CI) & Estimate (95\% CI) & Estimate (95\% CI) \\
\hline 'full' 450k/27k & $-0.28(-1.03,0.47)$ & $1.02(0.96,1.09)$ & $7.02(6.25,7.93)$ & $0.85(0.84,0.87)$ \\
'reduced' 450k/27k & $-0.32(-1.09,0.45)$ & $0.88(0.81,0.94)$ & $3.18(2.40,3.95)$ & $0.86(0.85,0.88)$ \\
\hline
\end{tabular}

Regressions of DNAm age on chronological age within the full and reduced datasets, within each age group, reveal further age-dependent differences (Table 3). Among those $<20 \mathrm{y}$,

228 the slope in the reduced datasets is shallower and significantly differ (t-test, $\mathrm{p}=0.002)$ when

229 compared with the full dataset, while the intercepts do not differ (t-test, $\mathrm{p}=0.94)$. Among those

$230 \geq 20$ years, the slopes do not differ significantly (t-test, $p=0.84$ ), but the underestimation of

231 DNA methylation age by the reduced data, as compared to the full data, is $3.84 \mathrm{y}$ (t-test,

$232 \mathrm{p}<0.001)$ at the intercept.

234 If the underestimation of DNA methylation age within each dataset is systematic,

235 associations between DNAm age and clinical variable (or other variable of interest) in the

236 reduced and full 450k datasets should be similar. Given the differences in DNAm age estimation

237 for individuals age $<20$ y vs $\geq 20$ y (Table 3, Figure S1), we examined associations between age

238 acceleration and sex, (Table 4) in both age groups. Using DNAm age acceleration, the residuals

239 of age regressed on DNAm age, the effect estimates obtained in the full 450k data were not

240 significantly different from those obtained in the reduced 450k data in subjects aged 20 years or

241 more $(\mathrm{p}=0.87)$ nor in subjects $<20$ years $(\mathrm{p}=0.22)$. This finding did not differ when we used

242 epigenetic age in place of the age acceleration measure (not shown), and did not differ depending

243 on whether the data was derived from the $27 \mathrm{k}$ array or $450 \mathrm{k}$ array. Residual violin plots for 
244 regressions of epigenetic age on sex (Figure S2) show no large or systematic differences in the

245 distribution of epigenetic age residuals, further reinforcing the similarity of the regressions with

246 and without the removal of the 17 probes missing from the $850 \mathrm{k}$ platform.

Table 4. Regressions of age acceleration on sex for CATHGEN450k and GEO datasets, using DNA methylation age calculated using the (full) $450 \mathrm{k}$ data and reduced $450 \mathrm{k}$ data.

250 Regressions were conducted for each dataset individually, and then in aggregate while 251 stratifying for chronological age $(<20 y$ and $\geq 20 y)$. P-values result from a $t$-test to compare the 252 slopes for regressions using the various DNAm ages.

\begin{tabular}{|c|c|c|c|c|}
\hline Dataset & $\begin{array}{l}\text { N (prop. } \\
\text { female) }\end{array}$ & $\begin{array}{l}\frac{\text { (Full) } 450 \mathrm{k} / 27 \mathrm{k} \text { data }}{\text { DNAm age }} \\
\text { Slope Est. }(95 \% \mathrm{CI})\end{array}$ & $\begin{array}{c}\frac{\text { Reduced } 450 \mathrm{k} / 27 \mathrm{k} \text { data }}{\text { DNAm age }} \\
\text { Slope Est. }(95 \% \mathrm{CI})\end{array}$ & $\frac{\frac{\text { Full vs. reduced }}{450 \mathrm{k} / 27 \mathrm{k} \text { data }}}{\text { p value }}$ \\
\hline Cathgen450k & $205(0.38)$ & $0.28(-1.31,1.87)$ & $0.39(-1.24,2.02)$ & 0.92 \\
\hline GSE20067 & $192(0.51)$ & $0.03(-1.64,1.7)$ & $0.12(-1.55,1.8)$ & 0.94 \\
\hline GSE20242 & $50(0.74)$ & $-3.31(-17.88,11.27)$ & $-1.58(-16.68,13.51)$ & 0.87 \\
\hline GSE32149 & $48(0.52)$ & $1.89(-1.58,5.37)$ & $2.25(-1.45,5.96)$ & 0.89 \\
\hline GSE40279 & $656(0.52)$ & $1.41(0.46,2.36)$ & $1.39(0.45,2.33)$ & 0.98 \\
\hline GSE41037 & $720(0.38)$ & $1.25(0.53,1.97)$ & $1.04(0.36,1.73)$ & 0.68 \\
\hline GSE41169 & $95(0.29)$ & $-0.89(-2.57,0.78)$ & $-1.13(-2.72,0.46)$ & 0.84 \\
\hline GSE42861 & $689(0.71)$ & $0.17(-0.69,1.03)$ & $0.01(-0.85,0.87)$ & 0.80 \\
\hline less than 20y & $662(0.06)$ & $-0.6(-1.52,0.32)$ & $0.19(-0.69,1.08)$ & 0.22 \\
\hline $20 \mathrm{y}$ or older & $3,294(0.60)$ & $1.51(1.01,2.01)$ & $1.57(1.07,2.07)$ & 0.87 \\
\hline
\end{tabular}

\section{Discussion}

255 Estimation of DNAm age is a methylation array dependent procedure, in so much as

256 differing arrays may not have all probes used to develop the DNAm age estimator. Use of the

257 epigenetic clock to estimate DNAm age from data generated from the Illumina MethylationEPIC

258 array is likely to produce substantial underestimation of DNAm age, relative to the DNAm age

259 estimated with the Illumina 450K array. A 3.3-year and 5-year increased DNAm age using the

260 Horvath epigenetic clock has been associated with an increase of 10 body mass index units (19)

261 and a 16\% increase in mortality (20), respectively. Thus, observed underestimations, in the range

262 of 4 years, could cause substantial mis-estimations of mortality and obesity risk based on the 
measured DNAm age if array differences are not accounted for. Using age-adjusted residuals

264 (DNA methylation age acceleration) or adjusting for age when using $\triangle$ age (DNAm age -

265 chronological age) as a predictor since the correlation between chronological age and DNA

266 methylation age appears to be independent of array. Systematic differences due to array design

267 would alter the intercept in such models but not regression coefficients. Thus, regression models

268 will reflect highly concordant results across arrays, but this will not necessarily be reflected in

269 comparisons of absolute epigenetic aging differences with outcomes across methylation

270 platforms. Estimating epigenetic age on a "reduced" 450k dataset (i.e. using probes only

271 available on the 850k array) produced similar underestimation as observed when using the 850k

272 data, indicating that the observed underestimation is primarily driven by the missing probes

273 (Table 1), as opposed to technological differences between the 850k and the 450k arrays. This

274 might be expected given the fact that the probes used for the 850k array used the same chemistry

275 and color channels as previous probes.

This study employed many of the same publicly available GEO datasets used to develop

277 the 450k clock, allowing direct comparisons in datasets which have been previously shown to

278 estimate DNAm age well (1). We focused on blood, since that is the tissue for which the Horvath

279 epigenetic age estimator provides the most accurate and consistent associations, and in which the

280 Horvath DNAm age estimator has been most widely applied. Because CATHGEN 450k and

281 850k data were estimated on independent (i.e., non-overlapping) groups of individuals, direct

282 comparison of the underestimation of DNAm age within individuals was not possible. However,

283 the size of the CATHGEN datasets still offer the ability to compare these measures in the same

284 source population, and both datasets were similar in age and sex makeup (Table 1).

The Illumina MethylationEPIC array represents a substantial step forward in the genome-

286 wide assessment of DNA methylation. As DNA methylation array technology has progressed,

287 researchers may wish to combine epigenetic age derived from 450k/27/k and 850k data;

288 however, the deviation in DNAm age estimates among the array platform generations may

289 introduce error into subsequent analyses. Thus, care should be taken when using epigenetic

290 biomarkers, such as Horvath's clock, that were developed using 450k and 27k data, as they may

291 not be fully optimized for the Illumina MethylationEPIC array. 


\section{Author contributions:}

293 RD and CWC are responsible for conception and design. RD was responsible for collecting,

294 processing and analyzing the publicly available data. RD and LK carried out processing and 295 analyses for CATHGEN. KO, DDS, RBD and WC provided funding for the creation of the

296 epigenetic data for CATHGEN on the 850K platform, and provided guidance in design and

297 execution of analyses. CH, ERH, SG, SS and WK are responsible for recruiting and maintaining

298 the CATHGEN biorepository; they supplied both the demographic and epigenetic data for

299 CATHGEN on the 450k platform. All authors have been involved in the editing of this paper and

300 have reviewed the final draft.

301 Acknowledgements: The authors would like to thank Kristen Rappazzo for critiques of the

302 manuscript and analysis. 
304 1. Horvath S. DNA methylation age of human tissues and cell types. Genome Biol [Internet]. 305 2013;14:R115. Available from: http://genomebiology.com/2013/14/10/R115

2. Dhingra R, Nwanaji-Enwerem JC, Samet M, Ward-Caviness CK. DNA Methylation AgeEnvironmental Influences, Health Impacts, and Its Role in Environmental Epidemiology. Curr Environ Heal reports [Internet]. Current Environmental Health Reports; 2018; Available from: http://www.ncbi.nlm.nih.gov/pubmed/30047075

3. Pidsley R, Zotenko E, Peters TJ, Lawrence MG, Risbridger GP, Molloy P, et al. Critical evaluation of the Illumina MethylationEPIC BeadChip microarray for whole-genome DNA methylation profiling. Genome Biol [Internet]. Genome Biology; 2016;17(208):1-

4. Horvath S. DNA Methylation Age Calculator [Internet]. [cited 2017 Jan 1]. Available from: dnamage.genetics.ucla.edu

5. Kraus WE, Granger CB, Jr MHS, Donahue MP, Ginsburg GS, Hauser ER, et al. A Guide Trans Res. 2015;8:449-57.

6. R Development Core Team. R: A language and environment for statistical computing.

321 7. Teschendorff AE, Menon U, Gentry-Maharaj A, Ramus SJ, Weisenberger DJ, Shen H, et al. Age-dependent DNA methylation of genes that are suppressed in stem cells is a hallmark of cancer. Genome Res. 2010;20(4):440-6.

8. Song H, Ramus SJ, Tyrer J, Bolton KL, Gentry-Maharaj A, Wozniak E, et al. A genomewide association study identifies a new ovarian cancer susceptibility locus on 9p22.2. Nat Genet. 2009;41(9):996-1000.

9. Bell CG, Teschendorff AE, Rakyan VK, Maxwell AP, Beck S, Savage DA. Genome-wide DNA methylation analysis for diabetic nephropathy in type 1 diabetes mellitus. BMC Med Genomics [Internet]. 2010;3(1):33. Available from: http://bmcmedgenomics.biomedcentral.com/articles/10.1186/1755-8794-3-33 
331 10. Rakyan VK, Down TA, Maslau S, Andrew T, Yang TP, Beyan H, et al. Human agingassociated DNA hypermethylation occurs preferentially at bivalent chromatin domains. Genome Res. 2010;20(4):434-9.

11. Alisch RS, Barwick BG, Chopra P, Myrick LK, Satten GA, Conneely KN, et al. Ageassociated DNA methylation in pediatric populations Age-associated DNA methylation in pediatric populations. Genome Res. 2012;22:623-32.

12. Heyn H, Li N, Ferreira H, Moran S, Pisano D, Gomez A, et al. Distinct DNA methylomes of newborns and centenarians. PNAS. 2012;109(26):10522-7.

13. Harris RA, Nagy-Szakal D, Pedersen N, Opekun A, Bronsky J, Munkholm P, et al.

14. Reinius LE, Acevedo N, Joerink M, Pershagen G, Dahlén SE, Greco D, et al. Differential DNA methylation in purified human blood cells: Implications for cell lineage and studies on disease susceptibility. PLoS One. 2012;7(7):e41361.

15. Hannum G, Guinney J, Zhao L, Zhang L, Hughes G, Sadda S, et al. Genome-wide Methylation Profiles Reveal Quantitative Views of Human Aging Rates. Mol Cell. Elsevier Inc.; 2013;49(2):359-67.

16. Horvath S, Zhang Y, Langfelder P, Kahn RS, Boks MPM, Eijk K Van, et al. Aging effects

17. Liu Y, Aryee MJ, Padyukov L, Fallin MD, Hesselberg E, Runarsson A, et al. Epigenomewide association data implicate DNA methylation as an intermediary of genetic risk in rheumatoid arthritis. Nat Biotechnol. 2013;31(2):142-7.

18. Heyn H, Moran S, Esteller M. Aberrant DNA methylation profiles in the premature aging 358 disorders Hutchinson-Gilford Progeria and Werner Syndrome. Epigenetics. 2013;8(1):2833. 
19. Horvath S, Erhart W, Brosch M, Ammerpohl O, Schönfels W Von, Ahrens M, et al.

Obesity accelerates epigenetic aging of human liver. PNAS [Internet].

20. Marioni RE, Shah S, McRae AF, Chen BH, Colicino E, Harris SE, et al. DNA methylation age of blood predicts all-cause mortality in later life. Genome Biol [Internet].

Table S1. Summary of GEO datasets.

Figure S1. Plot of reduced 450k DNA methylation age by $450 k$ data DNA methylation age in CATHGEN 450k data and the publicly available datasets for (a) all observations, (b) those < 20 years of age, and (c) those $\geq 20$ years of age. As can be seen across the plots, although the slope between the full and reduced DNA methylation age differs between the two age groups the overall correlation remains high.

375 Figure $S 2$. Violin plots of residuals by sex, from regression of DNA methylation age acceleration on sex for 450k data, reduced 450k data, in the CATHGEN 450k and publicly available $\mathbf{G E O}$ datasets. The distribution of residuals from the regression of age acceleration on sex is the same even after removing the 17 probes, indicating that regressions using age acceleration from the reduced $450 \mathrm{k}$ data (which underestimates DNA methylation age) remain 
CATHGEN 450k reduced, 450k clock: $y=0.76 x+12.84$

bioRxiv preprint doi: https://doi.org/10.1101/466045; this version posted November 8, 2018. The copyright holder for this preprint (which was not certified by peer review) is the author/funder. This article is a US Government work. It is not subject to copyright under 17 USC 105 and is
also made available for use under a CCO license.

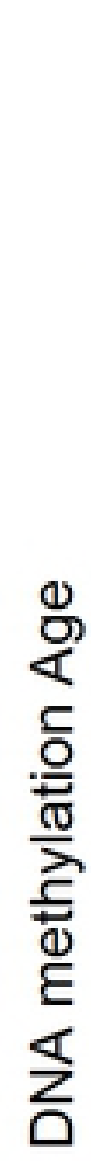
not certified by peer review) is the author/funder. This article is a US Government work. It is not subr
also made available for use under a CCO license.

$\frac{0}{2}$
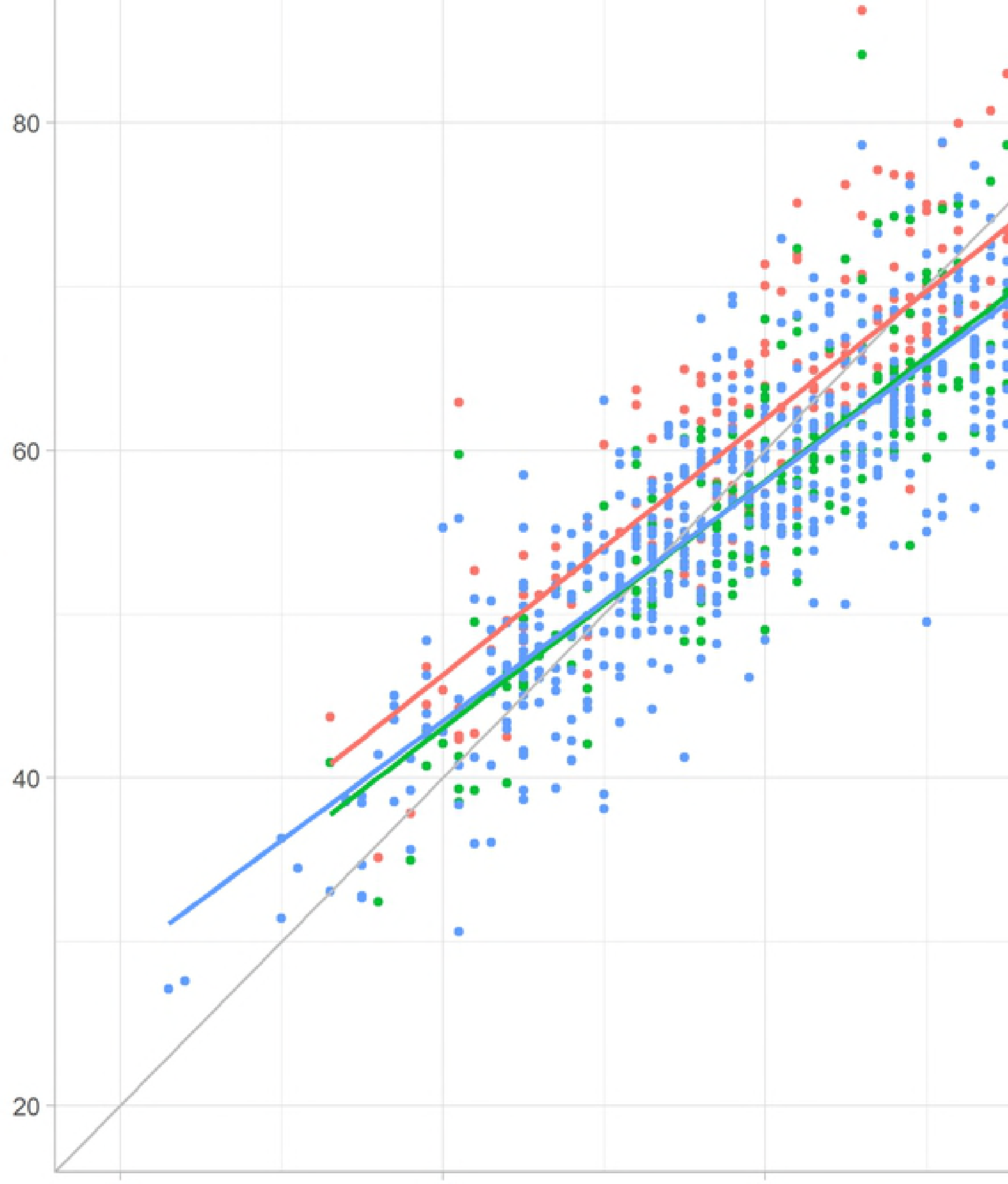
